\title{
Téoros
}

Revue de recherche en tourisme

\section{Bromont, de la vision à la réalité}

\section{Sylvie Vandal}

Volume 16, numéro 1, printemps 1997

La région touristique de l'Estrie / Cantons-de-l'Est

URI : https://id.erudit.org/iderudit/1074933ar

DOI : https://doi.org/10.7202/1074933ar

Aller au sommaire du numéro

\section{Éditeur(s)}

Université du Québec à Montréal

\section{ISSN}

0712-8657 (imprimé)

1923-2705 (numérique)

Découvrir la revue

\section{Citer ce document}

Vandal, S. (1997). Bromont, de la vision à la réalité. Téoros, 16(1), 69-71. https://doi.org/10.7202/1074933ar d'utilisation que vous pouvez consulter en ligne.

https://apropos.erudit.org/fr/usagers/politique-dutilisation/ 


\section{BROMONT, DE LA VISION À LA RÉALITÉ}

Sylvie Vandal, directrice-général Tourisme Bromont

Lhistoire de Bromont, si courte soit-elle, ne manque certainement pas d'effervescence et d'évenements. Cette ville aujourd'hui composée d'une population de cinq mille habi= tants est neconnue conme une importante destimation touristique de la région des Cantons-de-l'Est. Que ce soit par sa station touristique quatre saisons, par ses quatre parcours de golf par son centre de plein air ou par ses multiples choix d'hebergement, Bromont s'avere a theure actuelle une destination de choix tant pour les vacanciers que pour les gens d'affaines. Le nombre de visiteurs sur whe base annuelle oscille d'ailleurs autour de huit cent mille. Ce résultat découle de plusieurs années de planification et de réalisation appuyé fortement du dynamisme de ses batisseurs.
II y a un peu plus de trente ans, Bromont n'ctait qu"une idéce. Aujourd'hui blottie au pied du mont Brome et s'étendant dans la vallée de la rivière Yamaska, la ville de Bromont présente le coup d'oeil d" une localité bien planifiée. Cette planification s'est basée sur l'annexion d'une partie du territoire et sur la subdivision de ce demier. En effet, en annexant sous une mểme juridiction le Mont Brome, le village de West-Shefford, le village d'Adamsville jusqu"aux limites de West
Shefford ainsi qu'une partie des cantons de Shefford et de Brome, on se retrouvait avec une municipalité pouvant offrir à la fois de grands espaces et pour le développement industriel et pour le développement récréo-touristique. Des 5200 acres du depart, Bromont se compose aujourd'hui de plus de 33000 acres. C"est ainsi que tout le territoire a étét partagé en zones résidentielles, commerciales, industrielles et récréo-touristiques, le tout intercalé d'espaces verts. Chaque

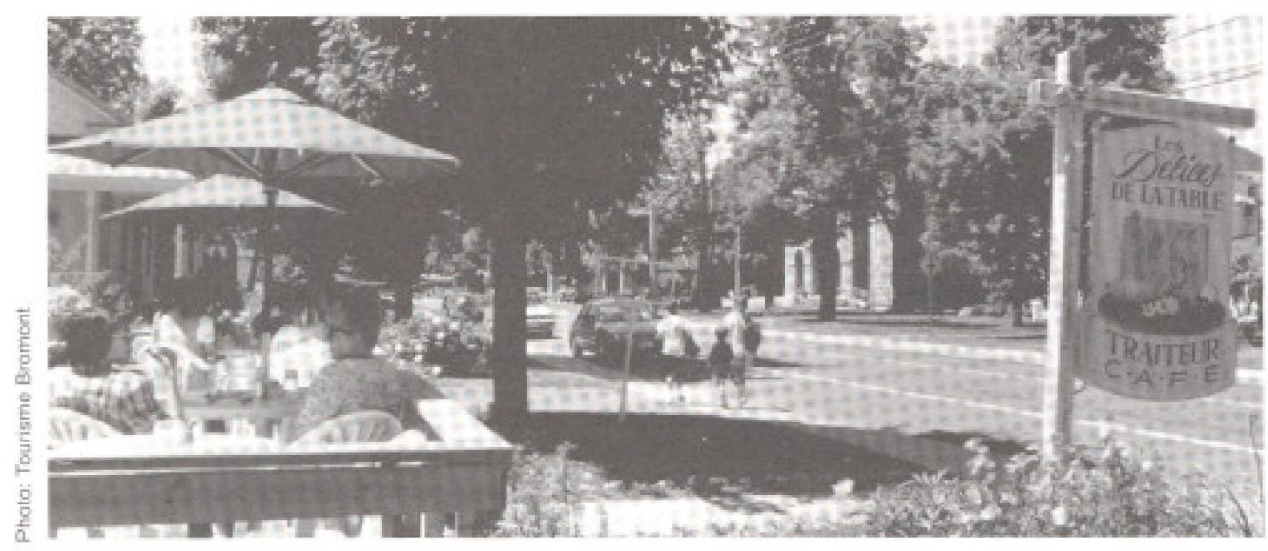

secteur occupe un endroit stratégique assurant un développement urbain harmonieux. Le plan de la ville réserve d'ailleurs une place de choix aux activités récréo-touristiques et ce, tant pour la population que pour les visiteurs.

Acronyme du nom Brome et du mot montagne, Bromont a recu ses lettres de noblesse le 18 juin 1964 du gouvernement Lesage et cela, malgré le fait que déjà à cette époque, ce demier favorisait la fusion et l'annexion de municipalitếs. Cette jeune ville fut fondée par un groupe d'entrepreneurs québecois dont les principaux artisans furent les frères Rolland et Germain Désourdy. Ce dernier fut d'ailleurs maire de la ville durant douze ans et demi.

Apres avoir voyagé un peu partout en Europe et aux Etats-Unis, les frères Désourdy avaient élaboré un tout nouveau concept urbain: la ville de l'an 2000. Privilégiée par sa localisation géographique, à proximité de Montréal et de la frontière américaine, et par sa topographie, Bromont constituait alors un emplacement de rêve pour développer le potentiel économique de la région. Bromont füt d'ailleurs une des rares municipalités à voir le jour à la fin des années 60 et à connaître de l'expansion.

Ces précurseurs se sont donc activés à doter la région d'infrastructures industrielles, récréatives et immobilières âfin d'assurer à Bromont un avenir industriel prometteur et une vocation touristique de premier plan. Bromont fut planifié et urbanisé selon des normes rigoureuses, axées sur la protection de l'environnement, de manière à respecter la tradi- 
tion et les habitudes de vie prévalantes dans les Cantons-de-l'Est. Bref, l'idée consistait à créer une ville, dotée de tous les services, tout en lui conservant un cachet campagnard et une qualité de vie recherchée. Bromont fut done conçu et planifié pour l'avenir. Le principal obstacle a été de vaincre les préjugés de la population avoisinante et de la convaincre du bien-fondé de cette ville nouvelle.

Le premier jalon important du développement mis de l'avant par les Désourdy fut d'ériger en 1968, un aéroport et ce, en plein champs!! Cet implant a favorisé l'installation de l'usine IBM en 1970. Par la suite, d'autres usines telles que G.E., Mitel, Hyundai, s'y sont installées créant par ricochet, un parc indutriel de haute technologie.

\section{LA STATION TOURISTIQUE DE BROMONT}

Parallèlement au développement de la ville, la famille Désourdy crée, en décembre 1964, la Station de ski Bromont. Le mont Brome connaissait une nouvelle vocation pour devenir une station de ski alors modeste avec trois pistes jouxtant le mont Soleil Bromont et ayant pour objectif de desservir la population locale et régionale. A l'été 1965, le golf Bromont, sis dans un paysage féérique, ouvre ses portes aux adeptes de ce sport.

En 1978, la relève de l'entreprise familiale se retrouve sous l'égide de Robert Désourdy, fils de Rolland. Dès lors, la réputation de la station est telle qu'elle sera l'hơte de la Coupe du monde de ski acrobatique en 1979. Reconnue alors pour son avant-gardisme, la Station de ski Bromont fut un des premiers centres de ski à offrir du ski de soirée et des pentes enneigées artificiellement en procédant à ces installations en 1980. D'où son importante popularité.

L'inauguration en 1984 du Parc Aquatique confere à la Station une vocation annuelle. En constante évolution, la Station touristique ouvrait, en 1985, de nouvelles pistes de ski tout en procédant à la réfection des pistes déjà existantes. De plus, $80 \%$ de la surface skiable sera alors éclairée. Au printemps de la même année. l'ajout de glissoires alpines au complexe d'été permettra d'étirer la saison jusqu"à lautomne.

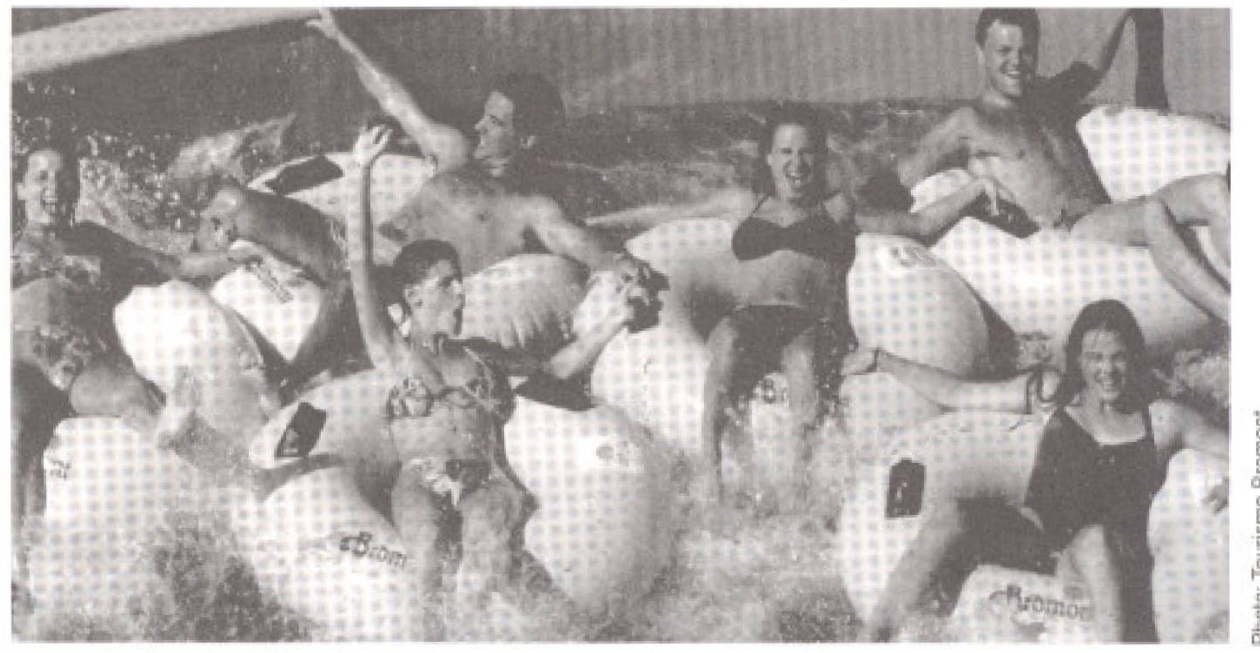

En 1986, le centre de ski reçoit la Coupe du Monde en ski alpin. Cet événement nécessitera de nombreuses améliorations techniques dont l'ajout d'un quadruple débrayable, la réfection et l'agrandissement du chalet de ski ainsi que l'acquisition du système d'enneigement York, entièrement informatisé. Ski Bromont est d'ailleurs la première station en Amérique du Nord à posséder ce système. A la fin des années 80, la Station touristique Bromont se voyait dotées de 24 pistes. 7 remontées et recevait quelque 300000 skieurs.

Le début des années 90 a vu nâtre à la station, une nouvelle discipline sportive: le vélo de montagne. Aujourd'hui plus de 100 kilomètres de pistes sont accessibles aux fanatiques de ce sport et les remontées mécaniques ont été adaptées pour la pratique de ce sport. La réputation de Bromont au niveau du vélo de montagne a acquis une notoriété internationale en recevant en 1992, le Championnat mondial de vélo de montagne. Le site est des plus appréciés par les athlètes pour la qualité de ses pistes et installations.

Dans un contexte où la population connaissait une augmentaion des heures de loisirs, la Station touristique de Bromont devint rapidement le moteur économique de la région et connaissait une fréquentation extraordinaire de ses installations.

Ainsi de façon directe et indirecte, ce succès a favorisé l'implantation d'installations récréatives multiples et variées. ainsi que d'infrastructures de services, hébergement et restauration. Cet essor démontre bien la justesse des prévisions des initiateur's de la municipalité.

\section{LE CENTRE ÉQUESTRE DE BROMONT}

C'est en parcourant la région à cheval que Rolland Desourdy, cavalier chevronné, a eu la vision de la ville de Bromont. Ferue des sports équestres, la famille Désourdy inaugurait en 1970, le Centre Équestre de Bromont. En 1976, les téléspectateurs du monde entier auront les yeux rivés sur Bromont qui est alors I'hôte des disciplines equestres des Jeux Olympiques d'été. Cet événement de grande envergure a permis à Bromont d'acquérir ses lettres de noblesse. De ce fait, la réputation acquise par Bromont $s^{*}$ est maintenue au fil des ans en recevant annuellement des compétitions équestres haut de gamme notamment I'International Bromont qui en est aujourd' hui à plus de trente éditions.

Témoignage éloquent de la beauté pittoresque de son territoire, le Centre Equestre sera le site des activités du Montreal Hunt Club, le plus ancien club de chasse à courre en Amérique du Nord. Reconnu comme un des centres les plus complets au Canada, le Centre Équestre de Bromont offre tous les services reliés à la pratique de ce sport et dispose également de 240 kilomètres d'allées cavalières en forêt et en montagne.

\section{LES COMPLEXES DE MAGASINS D'USINE}

Fort de la croissance phénoménale que connaissait aux États-Unis le concept des Factory Outlets, Bromont a vu poussé en son sein les complexes de magasins d'usine. Le début des années 90 fut matqué 
par un tourbillon roulant des millions de dollars d'investissement en construction de ces centres. En 1990, le premier complexe, les Versants de Bromont ouvrait ses portes et connaissait un tel succès commercial que moins de deux ans plus tard, ils procédaient à l'agrandissement de ces dernier via un investissement de 7,5 millions de dollars. Deux autres centres virent alors le jour, les Manufacturiers de Bromont et les Promenades Ma Maison. Ces derniers ont également réinvesti dans l'agrandissement de leur complexe et réinvesti une somme de $2,5 \mathrm{mil}=$ lions de dollars.

Cette période faste, ce succès prodigieux a cependant connu un déclin au cours des dernières années. Les Manufacturiers ont ferme leurs portes et les Versants de Bromont tentent actuellement de remonter la pente en donnant au complexe une nouvelle vocation. Quant aux Promenades Ma maison, elles tirent bien leur épingle du jeu.

\section{LES ÉVÉNEMENTS}

Quoique toute jeune, la ville de Bromont s'est distinguée en plus d'une occasion au niveau sportif intemational. A cet effet, le curriculum vitae de Bromont s'avère impressionnant:

1976: Jeux Olympiques, sports équestres Intarnational Bromont /Coupe du monde en sport équestre)

1978: Coupe du Monde de ski acrobatique International Bromont

1979: Etape de la Caupe du Monde en ski acrabatique

International Bromont

1984: Étape en ski alpin du circuit NOR-AM International Bromont

1985: Championnat canadien en ski alpin [catógorie juvenile\} Intemational Bromont

1986: Finale de la Coupe du monde en ski alpin Championnat Québécois sur route toutes catégories

International Bromont

1989: Champiónat de l'Est du Canada an vélo de montagne International Bromont

1990: Premier Championnat Canadien de vélo de montagne International Bromont

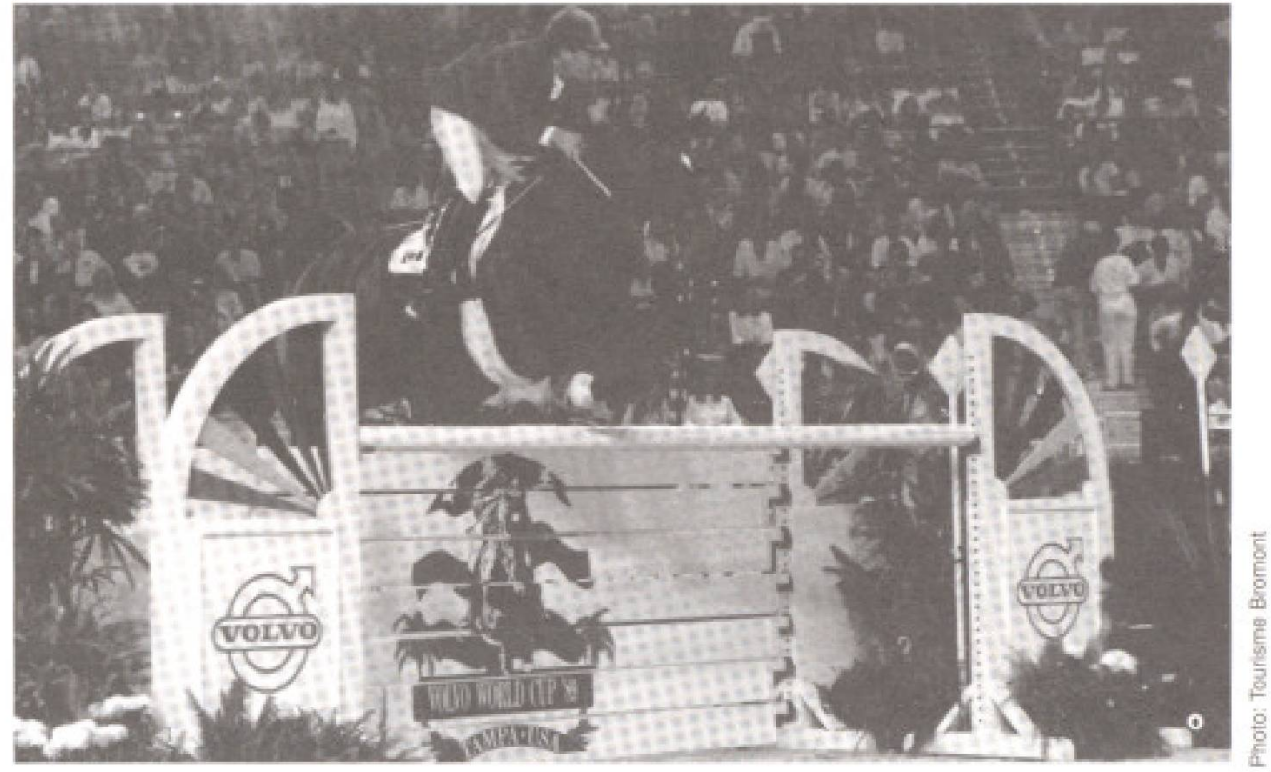

1991: Championnat canadien junior cyeliste sur route Championnat provincial Equestre de dressage Intemational Bromont

Journées Equestres de Bromont

|competitions áquestres|

1992. 3ieme Championnat du Monde de vèig de montagne

Championnat Nord-Americain

des jeunes cavalers

Championnat Canadien en ski alpin

Rallye international de camping \& caravaning

Finale au grand prix de Tourisme du Québoc: pour le vélo

Intemational Bromont

Journêes Écuestres de Bromont

1993: Coupe du mande véla de mantagne International Bromont

Journâces Équestres de Bromont

Symposium de peinture Fleurs en fête

1994: 1er Championnat du Québec, vélo de montagne Coupe du Québec, vélo de montagne International Bromont

Journéas Énuestres de Bromont

Symposium de peinture Fleurs en fête

1995. Coupe du Duébec, vélo de montagne Fitape du circuit NOR-AM en ski alpin International Bromont

Journées énuestres de Bromont

Symposium de peinture Fleurs en fệte

1966: Coupe du Monde de vélo de montagne Coupe du Quebec en ski de fond Concours complets (Compétitions équestres) International Bromont

Symposium de peinture Fleurs en fëte Exposition Internationale du Jouet Ancian Finale Coupe du Québec, vélo de montagne La nouvalle course Défi Route-Hors-route Centre National de dévaloppement cycliste

En mars 1988, un complexe hôtelier haut de gamme, le Chăteau Bromont, établis- sait ses pénates à Bromont bonifiant ainsi l'offre d'hébergement et ouvrant la porte a la clientèle de congres.

\section{EN GUISE DE CONCLUSION}

Foree nous est de constater qu'au fil des ans, l'offre touristique de Bromont s'est bonifiee. Plus de quatre-vingt entreprises touristiques ont pignon sur rue à Bromont et génère quelque mille emplois directs. Le tourisme constitue pour la localité la deuxième industrie en importance dans sa vie économique.

Privilegiée par sa localisation, par son cadre physique exceptionnel ainsi que par l'importance et l'envergure de son infrastructure récréo-touristique. Broont joue le rôle de porte d'entrée de la région touristique des Cantons-de-l'Est. Elle est d'ailleurs le premier point de chute pour accueillir le flux touristique en provenance de l'agglomération de Montréal. Depuis sa création, Bromont a connu un achalandage touristique soutenu. Quelque soit la saison, Bromont charme les amoureux de la nature et les sportifs.

Cette petite municipalite, sise dans les contreforts des Appalaches avait pour

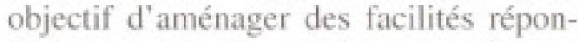
dant aux aspirations présentes et futures et de la population el de ses visiteurs. Aujourd'hui, ses fondateurs peuvent sans nul doute affirmer: mission accomplie! 